\title{
Magnetic resonance imaging in tracheal compression by the innominate artery
}

University Hospital, Ghent, Belgium, Department of Paediatrics F de Baets

$\mathrm{V}$ van de Weghe

\section{Department of} Radiology

A Smets

$\mathrm{K}$ Verstraete

Department of Cardiology Y Taeymans

Correspondence to: Dr F de Baets, University Hospital, Department of Paediatrics, De Pintelaan 185, 9000 Ghent, Belgium. Accepted 21 May 1993

F de Baets, $\mathrm{V}$ van de Weghe, A Smets, $\mathrm{K}$ Verstraete, $\mathrm{Y}$ Taeymans

\begin{abstract}
Tracheal compression by the innominate artery is frequently suspected in infants with noisy breathing and no obvious aortic arch anomalies or pulmonary artery sling. However the diagnosis is difficult to confirm. The findings after using magnetic resonance imaging in two infants with tracheal compression syndrome are presented.
\end{abstract}

(Arch Dis Child 1993; 69: 390-391)

Tracheal compression by mediastinal arterial vessels is a well recognised clinical syndrome in infants. ${ }^{12}$ These children have noisy breathing and airway obstruction and may experience severe respiratory distress including cyanotic spells. Tracheobronchoscopy is necessary to determine the location and degree of tracheal collapse, while the pulsating nature of the compression suggests a vascular origin. However tracheoscopy does not reveal the aetiology of the tracheal narrowing and evaluating the severity of obstruction is difficult. Fibreoptic bronchoscopy, under local anaesthesia, in an infant fighting the bronchoscope, can aggravate the impression of obstruction, while rigid bronchoscopy under general anaesthesia can underestimate its severity. Other diagnostic methods are necessary: tracheal compression by aortic arch anomalies or a pulmonary artery sling can be confirmed by angiography. Compression by the innominate artery is more difficult to prove. Imaging methods such as computed tomography, barium oesophagograms, and angiography combined with tracheography have been used. However the results are not very satisfactory. Magnetic resonance imaging (MRI) is recognised as a high quality imaging method in demonstrating mediastinal topography. Moreover it is a non-invasive technique, a further advantage in infants with respiratory distress. ${ }^{3}$ We present our findings in two infants after using MRI.

\section{Methods}

MRI was performed with a 1.5-T system (Magnetom 63 SP; Siemens Medical Systems, Erlangen, Germany). Children were sedated (oral chloral hydrate $20 \mathrm{mg} / \mathrm{kg}$ ) and placed in the head coil allowing a field of view of $300 \mathrm{~cm}$. The slice thickness was $2 \mathrm{~mm}$. The sequence used was a standard spin-echo with a TE of 15 msec and a TR equal to the RR interval. Synchronisation with the hard cycle was obtained using a Nonin pulse oximeter with a custom modified output. The alignment of the scanning plane was selected according to the longitudinal axis of the trachea. The total duration of the investigation was 30 minutes.

\section{Patients}

Two boys were born after 40 weeks' gestation of unrelated parents; in both cases pregnancy and delivery were normal.

At two months, case 1 was noted to have noisy breathing with recurrent respiratory infections. He was admitted to hospital several times because of short lasting cyanotic spells. Case 2 was admitted to hospital at the age of 3 months with bronchiolitis after which he experienced four bronchitic episodes. Noisy breathing was a persistent symptom and he failed to thrive.

Neither infant had stridor, but in expiration there was bulging of the suprasternal fossa. On auscultation there were inspiratory and expiratory rhonchi and expiration was prolonged. Arterial blood gases, peripheral blood examination, electrocardiography, and echocardiography were normal. A 24 hour oesophageal $\mathrm{pH}$ study showed multiple and prolonged episodes of reflux.

A barium oesophagogram in case 1 did not show any abnormal impression. Lateral chest radiography revealed slight compression of the anterior wall of the trachea in its lower third. Polysomnography showed multiple and prolonged obstructive apnoeic episodes without desaturation. At fibreoptic bronchoscopy severe compression of the left anterolateral part of the distal third of the trachea was seen. Compression of the trachea by the innominate artery was confirmed by MRI (fig 1).

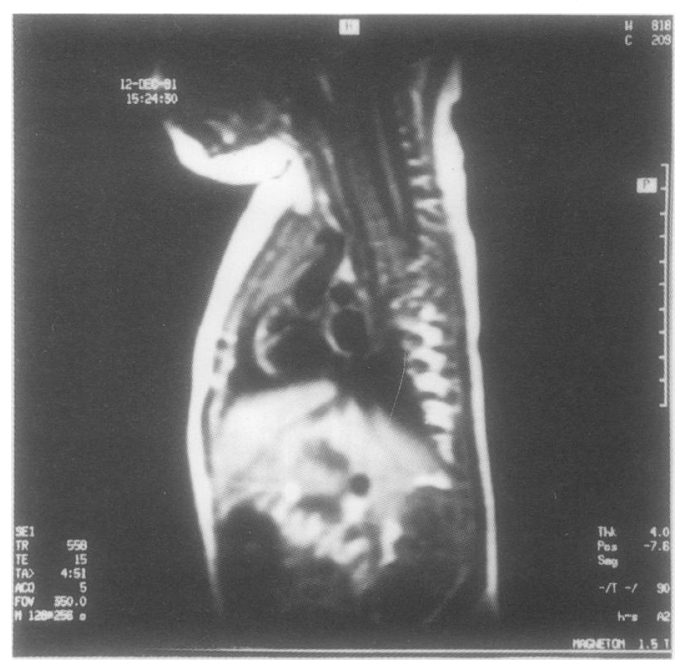

Figure 1 MRI scan showing tracheal compression by the innominate artery in case 1. 


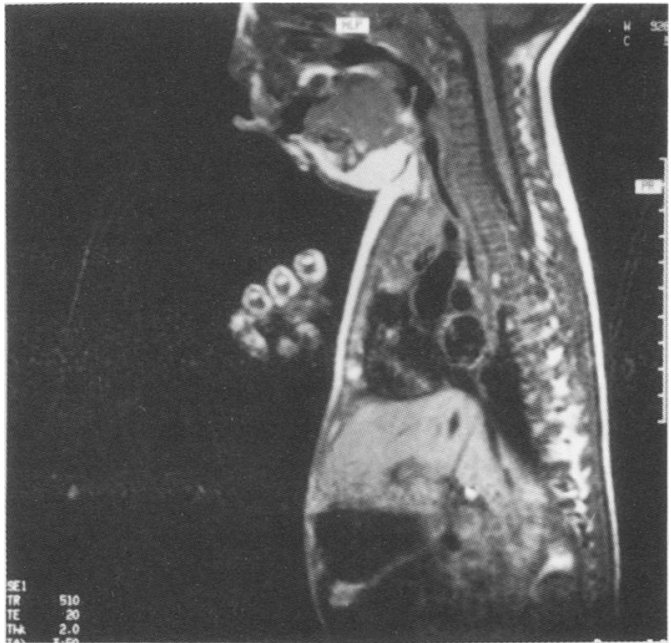

Figure 2 MRI scan showing tracheal compression by the innominate artery in case 2.

Lateral chest radiography in case 2 revealed a narrow intrathoracic trachea suggestive of tracheomalacia. Polysomnography showed multiple obstructive apnoeic episodes, prolonged expiration, and nocturnal oxygen desaturation (mean value: $87 \%$, range $81 \cdot 2 \%-92 \cdot 4 \%$ ). At fibreoptic bronchoscopy intrathoracic and extrathoracic tracheomalacia was seen. MRI demonstrated localised compression of the trachea by the innominate artery (fig 2).

Both infants were treated for gastrooesophageal reflux and an oral anticholinergic drug was given. The general practitioner was advised to treat respiratory infections with antibiotics. The final clinical outcome was favourable in both boys.

\section{Discussion}

While bronchoscopy revealed tracheomalacia in the second child, MRI surprisingly showed localised compression by the innominate artery. The image was probably obtained during inspiration as the trachea appeared a normal shape. However in this infant, it is not clear whether the innominate artery compression is the primary phenomenon with secondary tracheomalacia or whether it is secondary to pre-existing tracheomalacia.

In both patients, tracheal obstruction by the innominate artery was clearly shown by MRI. In these infants the innominate artery arises more to the left and consequently more posteriorly from the aortic arch, in such a manner as to pass over and compress the trachea. Although on MRI the obstruction seemed severe, both patients were treated conservatively. Their clinical outcome was favourable. Mustard et al reported successful results with medical treatment in $86 \%$ of 285 patients over a 16 year period. ${ }^{1}$ As is obvious from our patients the severity of compression found by MRI is not an indication for surgery. We do not agree with Vogl et al who advised aortopexy in all patients with innominate artery compression syndrome. ${ }^{3}$ Moreover, Clevenger et al reported recurrent respiratory distress after aortopexy in $50 \%$ of children with vascular compression of the trachea and major postoperative complications in $83 \%{ }^{4}$

We conclude that MRI is a valuable, noninvasive technique to demonstrate tracheal compression by the innominate artery. However MRI does not evaluate the need for surgical treatment.

1 Mustard WT, Bayliss CE, Fearon B, et al. Tracheal compression by the innominate artery in children. Ann compression by the innom
Thorac Surg 1969; 8: 312-9.

2 Gross RE, Neuhauser EB. Compression of the trachea by an anomalous innominate artery. An operation for its relief Am $\mathcal{F}$ Dis Child 1948; 75: 570-4

3 Vogl T, Wilimzig C, Hofman U, Hofman D, Dresel S, Lissner J. MRI in tracheal stenosis by innominate artery in children. Pediatr Radiol 1991; 21: 89-93.

4 Clevenger FW, Othersen HB, Smith CD. Relief of trachea compression by aortopexy. Ann Thorac Surg 1990; 50: 524-9. 